\title{
Prevalência e fatores de risco da mordida aberta anterior na dentadura decídua completa em pré-escolares na cidade de Natal/RN
}

Raniere Luiz dos Santos Sousa*, Rejane Bezerra de Lima**, Cícero Florêncio Filho***, Kênio Costa de Lima****, Alexandre Magno de Negreiros Diógenes*

\section{Resumo}

Objetivo: estimar a prevalência da mordida aberta anterior na dentadura decídua completa em crianças pré-escolares da cidade de Natal/RN e a associação desta a alguns fatores de risco. Metodologia: este estudo caracterizou-se por ser do tipo transversal e utilizou como elementos amostrais 366 indivíduos na idade de 5 anos na fase de dentadura decídua completa. A mordida aberta anterior, variável dependente, foi categorizada em presente e ausente. No que se refere às variáveis independentes estas foram divididas em: socioeconômicodemográficas e fatores extrínsecos. Através de um questionário fechado e estruturado, foram obtidas informações a respeito das variáveis socioeconômicodemográficas e os seguintes fatores extrínsecos: tipo de aleitamento, tempo de aleitamento materno exclusivo, tempo de aleitamento artificial, hábito e tempo de hábito. Através do exame clínico, foram obtidos os dados relativos ao padrão facial, considerado como fator extrínseco, e à variável dependente - mordida aberta anterior. A associação entre a mordida aberta anterior e as variáveis independentes foi obtida através do teste qui-quadrado com nível de significância de 5\%. Resultados e Conclusões: verificou-se associação significante entre a classe econômica, renda e a presença de hábito. A prevalência da mordida aberta anterior foi de $20,6 \%$ do total da amostra.

Palavras-chave: : Mordida aberta anterior. Dentição decídua. Fatores de risco.

\section{INTRODUÇÃO}

A mordida aberta é definida como uma deficiência no contato vertical normal entre os dentes antagonistas, podendo manifestar-se numa região limitada ou, mais raramente, em todo o arco dentário $^{27,41}$. Consiste em uma discrepância no sentido vertical, o que a torna mais difícil de ser corrigida e seus resultados finais mostram-se menos está- veis. Se a falta de contato dos dentes localiza-se na região de incisivos e/ou caninos, quando a oclusão está em relação cêntrica, esta passa a ser denominada de $\mathrm{MAA}^{15,27}$.

É consenso que a MAA ou a tendência à mordida aberta está entre os mais desafiantes tratamentos ${ }^{21}$. É uma das más oclusões de maior comprometimento estético-funcional, além das

* Especialista em Ortodontia pela ABO - RN

** Mestre em Odontologia Preventiva e Social da UFRN. Professora substituta da Disciplina Estágio Supervisionado CRUTAC - UFRN.

*** Especialista em Ortodontia pela UFPE. Professor do curso de Especialização em Ortodontia da ABO - RN. Mestrando em Ciências da Saúde da UFRN

**** Doutor em Ciências pela UFRJ. Professor do curso de Mestrado em Odontologia e Doutorado em Ciências da Saúde da UFRN. 
alterações dentárias e esqueléticas. Embora existam na literatura muitos relatos da sua autocorreção em uma alta porcentagem ${ }^{12,26,28,42,43}$, é fundamental que se conheçam os fatores de risco envolvidos na sua determinação, pois estes podem interferir no crescimento e desenvolvimento normais das estruturas faciais, modificando não somente a morfologia, mas também a função do sistema estomatognático.

Decorrente da sua complexidade e alto potencial em gerar problemas, o estudo da MAA, ainda na dentadura decídua, tem despertado muito interesse por parte de diversos autores, sendo a sua prevalência o tema mais abordado.

Estudando a prevalência da MAA, os autores Valente, Mussolino ${ }^{40}$; Paunio et al. ${ }^{30}$; Serra-Negra et $\mathrm{al}^{37}{ }^{37}$; Costa et $\mathrm{al}^{7}$; Milagre ${ }^{26}$; Forte, $\mathrm{Bosco}^{13}$; Mendes et al. ${ }^{25}$ e Emmerich et al. ${ }^{10}$ encontraram resultados significativos de MAA na dentadura decídua, variando de 23,32 a $55,4 \%$.

Alguns estudos transversais têm mostrado um decréscimo na freqüência de algumas más oclusões dos 3 aos $6 \operatorname{anos}^{12,21,26,28}$, dentre elas a MAA. A principal causa dessa diminuição deve-se às alterações de crescimento, modificações dentárias e diminuição da prevalência de hábitos deletérios com o avanço da idade.

O estudo realizado por Milagre ${ }^{26}$ na cidade de Natal - RN, com 742 crianças na fase de dentadura decídua completa, verificou a MAA como a má oclusão mais prevalente (30,3\%). A relação entre a presença de má oclusão e a idade foi a única que se mostrou significante tornando evidente a hipótese de que, com o aumento da idade durante a dentadura decídua, algumas oclusões tendem a passar de uma fase com características de anormalidade para uma oclusão normal.

Quanto aos fatores de risco, a despeito do grande número de estudos acerca das más oclusões existentes na literatura, há um número reduzido destes que visam determinar quais fatores estão relacionados à MAA.

Paunio et al. ${ }^{30}$ e Zuanon et al. ${ }^{44}$ destacaram a MAA como conseqüência de hábitos bucais viciosos, estando tais hábitos presentes em 25 e $61,34 \%$, respectivamente.

Segundo Katz ${ }^{19}$, o hábito de sucção não-nutritiva esteve fortemente associado com o desenvolvimento de má oclusão, sendo a MAA a mais prevalente neste estudo. Forte e Bosco ${ }^{13}$ verificaram a relação da MAA e os hábitos de sucção não-nutritiva em crianças na fase da dentadura decídua e concluíram que tais hábitos estiveram relacionados ao desenvolvimento desta má oclusão.

Serra-Negra et al. ${ }^{37}$ realizaram um estudo relacionando aleitamento natural, hábitos bucais e más oclusões. Concluíram que há uma associação entre o aleitamento natural e a não instalação de hábitos bucais viciosos e constataram, também, que a associação entre os hábitos bucais e as más oclusões foi significativa, sendo a MAA a má oclusão mais prevalente.

Alguns estudos têm apontado as condições estruturais da vida, como classe social ao nascimento do indivíduo, as condições econômicas, o tempo de freqüência à escola e a época de inserção no mercado de trabalho, como fatores que fortemente influenciam nas condições de saúde na vida adulta $^{3,34}$. Em se tratando das más oclusões, apesar de alguns fatores de risco serem apontados como causadores destes problemas, ainda não está bem definida como a relação entre eles ocorre, sugerindo que novas discussões devem ser realizadas para se compreender de maneira mais aprofundada o aumento da prevalência destes problemas ${ }^{31}$.

Diante do exposto, este trabalho objetivou estimar a prevalência da MAA na dentadura decídua completa e a associação desta a alguns fatores de risco em pré-escolares da cidade de Natal/RN.

\section{MATERIAL E MÉTODOS}

Este estudo foi realizado na cidade de Natal, capital do estado do Rio Grande do Norte (RN), no período de março de 2004 a janeiro de 2005. Caracterizou-se como um estudo do tipo transversal, sendo realizado na fase da dentadura decídua 
completa e, portanto, permitindo verificar a prevalência da MAA, bem como as principais variáveis que podem interferir para o surgimento desta.

Inicialmente, o projeto foi submetido à apreciação pelo Comitê de Ética em Pesquisa da Universidade Federal do Rio Grande do Norte (UFRN), sendo aprovado. Previamente ao estudo propriamente dito, foi realizado um estudo piloto com o objetivo de determinar o tamanho da amostra a ser incluída nesta pesquisa. Para tanto, foram sorteadas seis escolas, a partir da lista das escolas fornecida pela Secretaria de Educação do Estado do Rio Grande do Norte, com crianças na idade de 5 anos.

Anteriormente ao estudo piloto, foi realizada a calibração dos dois examinadores. Foi utilizado o coeficiente Kappa para medir a reprodutibilidade diagnóstica dos examinadores. $\mathrm{O}$ valor obtido foi de 0,866 que, segundo Landis e Koch ${ }^{23}$, representa uma ótima concordância.

\section{População do estudo}

As unidades amostrais primárias foram sorteadas a partir do cadastro do ano de 2004 dos estabelecimentos de ensino, registrados na Secretaria de Educação do Estado do Rio Grande do Norte. Previamente, foi realizada a exclusão de 31 estabelecimentos, pois estes eram especializados em crianças portadoras de algum tipo de deficiência, ou não possuíam a faixa etária de interesse, restando 344 unidades. Participaram, na condição de unidades amostrais, estabelecimentos de ensino de todas as 4 regiões da cidade. Nessas unidades foram obtidos elementos amostrais com idade de 5 anos de ambos os gêneros.

$\mathrm{Na}$ literatura ${ }^{33}$ indica-se o estabelecimento de um número (entre 20 e 30) que permita uma certa dispersão dessas unidades com a obtenção de um número razoável de elementos amostrais em cada uma delas. Em nosso estudo, foi utilizado um número de 20 unidades amostrais, identificados pela técnica de sorteio ponderado (grandeza utilizada = número de alunos da escola).
Foram incluídas, na condição de elementos amostrais, crianças com idade de 5 anos que estivessem na fase de dentadura decídua completa. As crianças nessa faixa etária que não se encontravam na fase de dentadura decídua foram excluídas do estudo.

O tamanho da amostra foi calculado com base no estudo piloto previamente descrito, tendo em vista a presença/ausência de más oclusões.

Como se trata de uma amostragem por conglomerados em múltiplos estágios, o número obtido foi posteriormente multiplicado por 2 , correspondente ao efeito do desenho - DEF (design effect), considerando que houve uma pré-estratificação (escola e aluno). Ao resultado encontrado foi acrescido 20\%, correspondente às possíveis perdas.

Dessa forma, o tamanho final da amostra para a dentadura decídua foi de 183 indivíduos/escola, totalizando, portanto, 366 indivíduos.

\section{Alocação dos elementos amostrais}

Após a identificação dos estabelecimentos de ensino que compuseram a amostra, foi realizado o sorteio dos elementos amostrais. A técnica utilizada para esse fim foi a da amostra casual sistemática. Para isso, foi realizada a junção das listas de alunos de cada escola, compondo uma relação única de todos os alunos e, em seguida, foi feita uma numeração seqüencial de todas as crianças da relação, até ser obtido o número total de alunos na faixa etária de interesse.

O valor total encontrado foi dividido pelo tamanho da amostra (determinado no estudo piloto) e, assim, foi obtido o intervalo amostral utilizado no processo de sorteio das crianças.

Após a definição da amostra, a fase subseqüente foi a localização dos responsáveis para a solicitação da autorização para realização dos exames.

\section{Variáveis do estudo}

A mordida aberta anterior (desfecho) foi categorizada em presente e ausente. No que se refere 
às variáveis independentes, estas foram divididas em: socioeconômicodemográficas e fatores extrínsecos.

Em relação às socioeconômicodemográficas foram analisadas as seguintes variáveis: gênero, etnia $^{18}$, tipo de escola, classificação econômica ${ }^{1}$ e renda familiar.

Em relação aos fatores extrínsecos, foram avaliados o padrão facial, tempo e tipo de aleitamento e a presença e o tempo de hábitos.

\section{Coleta de dados}

Através de um questionário fechado e estruturado, foram obtidas informações a respeito das seguintes variáveis socioeconômicodemográficas e das variáveis independentes: tipo de aleitamento, tempo de aleitamento materno exclusivo, tempo de aleitamento artificial, hábito e tempo de hábito.

Os dados clínicos deste estudo corresponderam ao padrão facial, pertencente aos fatores extrínsecos, e à variável dependente - MAA. Para a avaliação destes dados foram utilizadas espátulas de madeira descartáveis para afastamento de tecidos moles. Também foi utilizado um paquímetro analógico Mitutoyo (Japão), com precisão de $0,01 \mathrm{~mm}$, para medir a altura facial (distância do ponto Násio ao Gnátio) e largura bizigomática (distância entre os pontos do zigoma) dos indivíduos examinados. Para o diagnóstico da mordida aberta anterior foi considerado um trespasse vertical negativo superior a $1 \mathrm{~mm}$, medido utilizandose uma régua milimetrada.

\section{Analise dos dados}

Inicialmente foi realizada uma análise descritiva através de proporções (distribuição das freqüências - freqüência absoluta e relativa), correspondente a cada variável do presente estudo. Ademais, a fim de realizar inferências, foram construídas tabelas de contigência (2x2), a fim de testar a presença da relação, bem como a sua magnitude entre a presença de mordida aberta ante- rior e as variáveis independentes. Para quantificar a magnitude do efeito foram calculados, além do teste do Qui-quadrado com correção de Yates e a respectiva significância estatística, as razões de chance de ocorrência da variável dependente (resposta) (Odds ratio) e os respectivos intervalos de confiança.

Quando a freqüência observada, nas caselas da tabela foi $<5$, optou-se pelo teste exato de Fisher para o mesmo fim (relação entre as variáveis, bem como magnitude) com respectiva Odds ratio e intervalo de confiança.

\section{RESULTADOS}

Do total da amostra dos escolares em fase de dentadura decídua que estudavam em escola pública ( $\mathrm{n}=183)$, foram obtidas 172 observações, havendo uma perda de $6 \%(\mathrm{n}=11)$, devida à recusa dos responsáveis em participarem do estudo. No que se refere aos indivíduos das escolas privadas, obtiveram-se 154 exames, havendo uma perda de $15,8 \%$ ( $n=29)$, ocasionada, também, pela não autorização dos exames. Estas perdas ocorreram dentro do esperado, ou seja, em torno de $20 \%$, valor já incluído no cálculo do tamanho da amostra.

Quanto à caracterização da amostra, verificou-se uma predominância do gênero feminino $(59,8 \%)$ em relação ao masculino $(40,20 \%)$. Quanto à etnia, houve uma predominância dos pardos na população estudada $(69,30 \%)$, em relação aos negros (16\%) e brancos (14,7\%). No que se refere à distribuição dos examinados em relação ao tipo de escola, houve um predomínio de crianças pertencentes à rede pública de ensino $(52,8 \%)$ em relação à rede privada $(47,2 \%)$.

Quando se observou a distribuição das classes, verificou-se uma predominância das classes econômicas D e C, como observado no gráfico 1 . O quadro socioeconômico descrito nos remete às características da população brasileira, onde há um predomínio, de acordo com o Levantamento Socioeconômico ${ }^{1}$, das classes econômicas C (31\%) e D (33\%). Tais resultados também foram encon- 


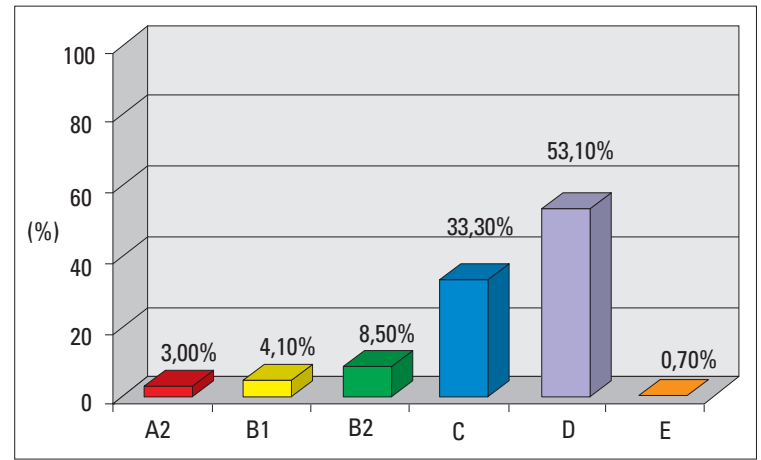

GRÁFICO 1 - Distribuição percentual entre as diferentes classes sociais da pesquisa.

trados neste estudo, refletindo que a população examinada foi similar à brasileira.

Nesse estudo observou-se que, no que se refere à renda familiar, mais de $50 \%$ da população apresentou renda que não ultrapassou quatro salários mínimos (SM).

No que se refere aos fatores extrínsecos, quando da avaliação do padrão facial neste estudo, observou-se uma predominância para o tipo mesofacial $(70,9 \%)$, em relação ao dolicofacial $(16,6 \%)$ e braquifacial $(12,5 \%)$.

Um outro fator extrínseco avaliado foi o tipo de aleitamento materno, sendo o materno exclusivo (AME) o que apresentou maior expressão dentro do universo analisado $(85,8 \%)$.

No que se refere ao tempo de aleitamento, observou-se que 70,1\% do aleitamento materno exclusivo (AME) foi realizado por um período igual ou superior aos 6 meses de idade. Observando-se o tempo de aleitamento artificial, verificou-se uma maior prevalência para o tempo igual ou superior aos 6 meses de idade $(66,7 \%)$.

No que se refere à variável "possuiu hábito", foi encontrado um percentual de $27,8 \%$ que possuíam algum hábito.

Quanto ao tempo de realização do hábito, o percentual foi de $69,1 \%$ com duração acima dos três anos de idade.

Para a população do estudo que apresentou as características anteriormente citadas, observou-se uma prevalência de mordida aberta anterior de 20,6\%.

\section{Fatores de risco $\mathrm{x}$ mordida aberta anterior}

Para análise das possíveis associações entre a variável dependente e as independentes foram realizadas algumas recategorizações, observandose a literatura e respeitando-se os marcos teóricos adotados. As variáveis recategorizadas foram, na verdade, dicotomizadas para melhor visualização do efeito da associação.

No que se refere à relação entre as variáveis socioeconômicodemográficas e o desfecho, mordida aberta anterior, verificou-se associação significante entre a classe econômica $(\mathrm{p}<0,0001)$ e renda $(\mathrm{p}=0,001)$, sendo as condições desfavoráveis e baixa renda fatores de proteção para o desenvolvimento de mordida aberta anterior. As demais variáveis, gênero $(p=0,170)$, etnia $(p=0,87)$ e escola $(\mathrm{p}=0,360)$, não apresentaram associação significante com a presença da mordida aberta anterior (Tab. 1).

Quando foi buscada a associação entre os fatores extrínsecos e a ocorrência de mordida aberta anterior, verificou-se associação apenas para a presença de hábito $(\mathrm{p}=0,0001)$. As demais variáveis, padrão facial $(p=0,29)$, tempo de hábito $(p=0,85)$, aleitamento durante a infância $(p=0,087)$, tempo de aleitamento materno $(\mathrm{p}=1,00)$ e tempo de aleitamento artificial $(p=0,927)$, não apresentaram associação significante com a presença da mordida aberta anterior (Tab. 1).

\section{DISCUSSÃO}

Para um maior aprofundamento das questões acerca da saúde bucal, incluindo o tratamento de média e alta complexidade, bem como um planejamento adequado das ações no âmbito da prevenção, promoção e recuperação, faz-se necessário o conhecimento da distribuição das doenças bucais, bem como dos seus fatores de risco, para que métodos de prevenção e/ou intervenção possam ser adequadamente implantados.

Dessa forma, a realização deste estudo teve propósitos ímpares, especialmente no que se refere à população do município de Natal, onde há 
insuficiência de estudos acerca da mordida aberta anterior e seus fatores determinantes.

Tomando como base a prevalência, este estudo observou a mordida aberta anterior num percentual de 20,6\% dos indivíduos, valor inferior aos encontrados por Costa et al. ${ }^{7}$; Emmerich et al. ${ }^{10}$; Forte, Bosco ${ }^{13}$; Mendes et al. ${ }^{25}$; Milagre $^{26}$; Paunio et al. ${ }^{30}$; Serra-Negra et al. ${ }^{37}$; Valente e Mussolino ${ }^{40}$. Essa diferença, em relação à prevalência, pode ser explicada devido às características de cada população estudada como: fatores socioeconômicoculturais das populações, prevalência de hábitos de sucção não nutritiva, assim como pelos diferentes tamanhos das amostras ${ }^{13}$.

Mesmo havendo uma semelhança entre este estudo e o de Milagre $^{26}$ com relação à amostra estudada (dentadura decídua completa no município de Natal-RN) e quanto à metodologia empregada, houve uma diferença com relação à prevalência da mordida aberta anterior de 20,6\% para este estudo e de 30,3\% para o estudo de Milagre ${ }^{26}$. Esta diferença pode ser explicada pela faixa etária dos indivíduos. No estudo de Milagre ${ }^{26}$, a amostra foi composta por indivíduos entre 36 e 60 meses de idade, enquanto este estudo teve como amostra apenas indivíduos com 60 meses de idade e, assim,

Tabela 1 - Análise univariada da associação entre a mordida aberta anterior na dentadura decídua com as variáveis independentes estudadas.

\begin{tabular}{|c|c|c|c|c|c|c|}
\hline \multirow[b]{2}{*}{ variável } & \multirow[b]{2}{*}{ categoria } & \multicolumn{2}{|c|}{ MORDIDA ABERTA ANTERIOR } & \multirow[b]{2}{*}{$\mathbf{p}\left(X^{2}\right)$} & \multirow[b]{2}{*}{ odds ratio } & \multirow[b]{2}{*}{ IC $(95 \%)$} \\
\hline & & $\begin{array}{c}\text { presente } \\
n(\%)\end{array}$ & $\begin{array}{c}\text { ausente } \\
n(\%)\end{array}$ & & & \\
\hline \multicolumn{7}{|c|}{ socioeconômicodemográfico } \\
\hline \multirow{2}{*}{ gênero } & feminino & $45(23,1)$ & $150(76,9)$ & \multirow{2}{*}{0,17} & \multirow{2}{*}{1,49} & \multirow{2}{*}{$0,844-2,619$} \\
\hline & masculino & $22(16,8)$ & $109(83,2)$ & & & \\
\hline \multirow{2}{*}{ etnia } & pardo & $47(29,8)$ & $179(79,2)$ & \multirow{2}{*}{0,87} & \multirow{2}{*}{1,05} & \multirow{2}{*}{$0,585-1,887$} \\
\hline & branco/negro & $20(20,0)$ & $80(80,0)$ & & & \\
\hline \multirow{2}{*}{ escola } & pública & $32(18,6)$ & $140(81,4)$ & \multirow{2}{*}{0,36} & \multirow{2}{*}{0,78} & \multirow{2}{*}{$0,454-1,331$} \\
\hline & privada & $35(22,7)$ & $119(77,3)$ & & & \\
\hline \multirow{2}{*}{ classe econômica } & desfavorável & $20(12,7)$ & $138(87,3)$ & \multirow{2}{*}{$<0,0001$} & \multirow{2}{*}{0,29} & \multirow{2}{*}{$0,163-0,528$} \\
\hline & favorável & $45(33,1)$ & $91(66,9)$ & & & \\
\hline \multirow{2}{*}{ renda } & $\leq 3 \mathrm{SM}$ & $24(15,0)$ & $136(85,0)$ & \multirow{2}{*}{0,001} & \multirow{2}{*}{0,39} & \multirow{2}{*}{$0,219-0,685$} \\
\hline & $>3 \mathrm{SM}$ & $41(31,3)$ & $90(68,7)$ & & & \\
\hline \multicolumn{7}{|c|}{ fatores extrínsecos } \\
\hline \multirow{2}{*}{ padrão facial } & dolico/braqui & $16(16,8)$ & $79(83,2)$ & \multirow{2}{*}{0,29} & \multirow{2}{*}{0,72} & \multirow{2}{*}{$0,384-1,330$} \\
\hline & mesofacial & $51(22,1)$ & $180(77,9)$ & & & \\
\hline \multirow{2}{*}{ hábitos } & possui & $45(55,6)$ & $36(44,4)$ & 0 & 1169 & $6186-22080$ \\
\hline & não possui & $20(9,7)$ & $187(90,3)$ & $-0,0001$ & & 0,100 \\
\hline temno de háh & $>3$ anos & $32(57,1)$ & $24(42,9)$ & 0.91 & 122 & \\
\hline 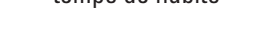 & $\leq 3$ anos & $13(52)$ & $12(48)$ & 0,00 & 1,20 & $0,470-0,1111$ \\
\hline aloitam duranto infância & artificial & $14(34,1)$ & $27(65,9)$ & 000 & & \\
\hline 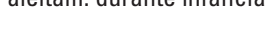 & ao Seio & $51(20,6)$ & $196(79,4)$ & 0,001 & 1,99 & $0,9 / 5-4,0 / 5$ \\
\hline tempo de aleitam. & $<6$ meses & $16(21,3)$ & $59(78,7)$ & 100 & 102 & $0526-1973$ \\
\hline materno & $\leq 6$ meses & $37(21)$ & $139(79)$ & 1,00 & 1,02 & $0,5<b-1,9 / 3$ \\
\hline tempo de aleitam. & $<6$ meses & $06(23,1)$ & $20(76,9)$ & 0927 & 0814 & $0271-2444$ \\
\hline artificial & $\leq 6$ meses & $14(26,9)$ & $38(73,1)$ & $0,0<1$ & 0,014 & $0,217-2,4+4$ \\
\hline
\end{tabular}


mais susceptíveis ao processo de auto-correção e, conseqüentemente, com menor prevalência da mordida aberta anterior.

$\mathrm{Na}$ literatura, a má oclusão mais prevalente encontrada na dentadura decídua tem sido a $\mathrm{MAA}^{4,12,24,29,39}$, que decresce, em termos de prevalência, com o avanço da idade $12,26,28,42,43$. A principal causa dessa diminuição, conforme citado anteriormente, deve-se a alterações de crescimento, modificações dentárias e diminuição da prevalência de hábitos deletérios com o avanço da idade.

De uma maneira geral, em relação aos fatores de risco socioeconômicodemográficos do presente estudo, após análise univariada, verificou-se que as principais variáveis relacionadas com a prevalência da mordida aberta anterior foram a renda e a classe econômica, sendo o nível socioeconômico desfavorável fator de proteção para o desenvolvimento desta má oclusão.

Essa relação pode ser justificada pela dificuldade de acesso, por parte desse grupo, à chupeta, principal fator de risco, segundo a literatura, implicado com o desenvolvimento da MAA. No entanto, mais estudos, de natureza longitudinal, devem ser conduzidos para a confirmação desta hipótese.

Contrariamente aos achados deste estudo, a literatura não aponta associação entre condições socioeconômicas e más oclusões na dentadura decídua ${ }^{20,31,38}$. No estudo realizado por Peres ${ }^{31}$, a hipótese de não-associação parece ser irrefutável, pois se trata de um estudo de coorte, diferentemente dos outros dois estudos seccionais ${ }^{20,38} \mathrm{e}$, portanto, os fatores de risco podem ser melhor observados e relacionados. No entanto, em relação à validade externa deste estudo, devem ser consideradas as particularidades da população estudada, pois o município de Pelotas/RS apresenta um dos melhores indicadores de condição social e de saúde do Brasil e, portanto, difere da amostra do presente estudo.

No que se refere à etnia, Tomita, Bijella e Franco $^{38}$, avaliando a oclusão de crianças na fase de dentadura decídua na cidade de Bauru-SP, não encontraram associação entre más oclusões e essa variável. Do mesmo modo, Frazão ${ }^{14}$ não observou essa relação em escolares em fase de dentadura decídua e permanente. Portanto, esses achados corroboram os dados desse estudo, uma vez que não foi encontrada associação entre mordida aberta anterior e etnia.

Estudo realizado por Infante ${ }^{17}$, nos EUA, com 735 crianças, em fase de dentadura decídua, revelou que há diferença no percentual das más oclusões, principalmente em relação à mordida cruzada posterior, entre brancos, negros e índios. Estudos nacionais ${ }^{14,26}$ revelaram que as diferenças étnicas não influenciaram na prevalência de más oclusões, sendo esses achados esperados para a população brasileira, uma vez que não são encontradas, com relativa freqüência, populações puras no Brasil.

Deve-se ressaltar que os estudos descritos acima não traduzem especificamente a relação entre mordida aberta anterior e etnia, mas de uma maneira geral apontam que não há associação entre a presença de más oclusões e essa variável. Portanto, mais estudos devem ser realizados para a verificação desta hipótese.

Uma relação comumente avaliada nos estudos é a associação entre gênero e prevalência de doença (ou agravos à saúde). No entanto, no presente estudo essa associação não foi significante. Este achado já era esperado, em conformidade com a literatura $^{14,20,31}$, cujos estudos não demonstraram diferenças na freqüência dos problemas oclusais entre os gêneros masculino e feminino.

Uma outra variável comumente avaliada é o tipo de estabelecimento de ensino, se público ou privado, a qual, no presente estudo, não mostrou associação com a presença de mordida aberta anterior.

No que se refere aos achados relativos aos fatores extrínsecos, particularmente em relação ao tipo e tempo de aleitamento, não houve associação significante com o desenvolvimento de mordida aberta anterior. Relação semelhante foi 
encontrada por Baldrighi et al. ${ }^{2}$, a despeito do percentual de crianças que receberam amamentação artificial, ser consideravelmente maior (73,33\%) do que deste estudo e apesar da autora avaliar 180 crianças, de 4 a 6 anos de idade, apenas de escolas da rede pública de Bauru.

Estudo retrospectivo realizado por Labbok e Hendershot ${ }^{22}$ verificou que crianças que utilizaram mamadeira tiveram 1,84 vezes mais chance de desenvolverem algum problema oclusal, quando comparadas com aquelas amamentadas naturalmente, independente da dentadura avaliada.

Davis e Bell ${ }^{8}$, em um estudo longitudinal, do nascimento aos 5 anos, com crianças de um serviço médico do Canadá, verificaram uma forte associação entre aleitamento artificial e alterações oclusais $(\mathrm{p}=0,006)$.

A relação entre o tempo de aleitamento materno exclusivo e o desenvolvimento de más oclusões, particularmente a mordida aberta anterior, foi avaliada em um estudo de coorte, do nascimento à primeira infância, no município de Pelotas/RS ${ }^{31}$. Nesse estudo, verificou-se que crianças que receberam aleitamento materno exclusivo por menos de 4 meses tiveram 3 vezes mais chance de desenvolverem más oclusões.

Como relatado, os estudos que indicaram uma associação significante entre tipo e tempo de aleitamento são investigações que avaliaram a criança desde o nascimento e, portanto, possuem evidências claras dessa associação, respeitando-se a localidade onde foram realizados.

No presente estudo, a natureza transversal provavelmente não possibilitou a verificação causa/ efeito da associação entre aleitamento e mordida aberta anterior. Um outro aspecto a ser considerado é o viés de memória, comumente encontrado nos estudos dessa natureza.

No que se refere à existência do hábito, após a realização da análise univariada, verificou-se um efeito significativo entre o hábito e a prevalência de mordida aberta anterior, apresentando as crianças que possuíam hábito 11,69 vezes mais chance de desenvolverem mordida aberta anterior.

A despeito de na literatura esta associação entre hábitos e más oclusões na dentadura decídua estar bem clara ${ }^{5,9,16,19,31,32,35,36,37,38}$, deve-se considerar que o desequilíbrio dentofacial nem sempre é o resultado de um determinado fator etiológico, mas o que existe é uma predisposição, a qual o hábito deletério apenas viria desencadear ou intensificar. Portanto, é a interação dos hábitos deletérios com os padrões faciais que determina sua influência na face, sendo a qualificação do hábito e as características individuais os fatores diferenciais ${ }^{6}$. Dessa forma, ao se verificar os resultados da análise univariada, percebe-se que o padrão facial não apresentou efeito significativo para o desenvolvimento da mordida aberta anterior.

Quanto ao padrão facial, devido à sua característica hereditária e, portanto, de difícil intervenção em nível público, algumas considerações são necessárias.

Segundo Enlow"11: "O crescimento craniofacial é um processo que requer íntimas relações morfogênicas entre todas as partes de tecidos duros e moles em crescimento, mudança e funcionamento que o compõem. Nenhuma é independente ou auto-suficiente".

Verifica-se que há uma relação estreita entre o padrão facial e as estruturas dos arcos dentários e, portanto, diversos fatores podem interferir e, dependendo da tendência de crescimento e desenvolvimento, acentuar ou predispor a ocorrência de más oclusões - é o caso dos hábitos.

Deve-se levar em consideração que o hábito não modifica nem desenvolve um tipo facial, porém o acentua. Partindo-se dessa hipótese, outras variáveis podem interferir no padrão facial, de maneira a desequilibrar sua harmonia e acentuar as más oclusões e, portanto, são essas variáveis que devem ser analisadas.

No que se refere ao presente estudo, embora poucos fatores de risco tenham mostrado significância, deve-se, primeiramente pensar na(s) causa(s) da alta prevalência da mordida aberta 
anterior na dentadura decídua. Levando-se em consideração a equação ortodôntica ("equação de Dockrell”, citada por Frazão ${ }^{14}$ ) tem-se que:

CAUSAS $\underset{\text { certo }}{\longrightarrow}$ TEMPO $\stackrel{\text { sobre }}{\longrightarrow}$ TECIDOS $\stackrel{\text { produzem }}{\longrightarrow}$ RESULTADOS

São esses os resultados (mordida aberta anterior) que o presente estudo ressalta. E, pensando mais uma vez na lógica do bem estar bio-psico-social do homem, enquanto ser integral, novos estudos devem ser conduzidos no sentido contrário ao da equação acima exposta, para tentar identificar as causas da mordida aberta anterior e a partir daí desenvolver medidas preventivas.

\section{CONCLUSÃO}

Conclui-se através deste estudo que a prevalência da mordida aberta anterior em crianças na idade de 5 anos e na dentadura decídua completa na cidade de Natal-RN apresentou um valor de $20,6 \%$, abaixo do encontrado na literatura, havendo associação significativa desta má oclusão apenas com a classe econômica e renda (variáveis socioeconômicodemográficas) e com a presença de hábitos (fatores extrínsecos).

Mais estudos necessitam ser realizados, no sentido de esclarecer melhor estas relações, para que medidas estratégicas possam ser melhor direcionadas e, com isso, ocorra uma diminuição desse problema, tanto no nível individual quanto no coletivo.

\title{
Prevalence and risk factors of anterior open bite in the complete deciduous dentition in pre-scholars'children who live in the city of Natal/RN
}

\begin{abstract}
Aim: The aim of this study was to determine the prevalence of the anterior open bite in the complete deciduous dentition of pre-scholars children who live in the city of Natal/RN, and the association of this malocclusion to possible risk factors. Methods: A transverse study was performed. Three hundred sixty six individuals, 5 yearsold, with complete deciduous dentition were examined. Anterior open bite, the dependent variable, was classified into present or absent. A number of independent variables were collected through a closed and structured questionary. These were classified into socio-economic-demographic and extrinsic factors. The extrinsic factors were breast-feeding type, time of exclusive maternal breast-feeding, time of artificial breast-feeding, presence of habit and habit duration. The determination of presence of open bite and the facial pattern (extrinsic factor) was done through clinical exams. The association between the anterior open bite and the independent variables was analysed through chi-square tests with a significance value of $5 \%$. Results and Conclusions: Significant association was found between the economic class, family income and the presence of habits. The prevalence of the anterior open bite was $20.6 \%$.
\end{abstract}

Key words: Anterior open bite. Deciduous dentition. Risk factors.

\section{REFERÊNCIAS}

1. ASSOCIAÇÃO NACIONAL DE EMPRESAS DE PESQUISA. Critério de Classificação Econômica Brasil. Levantamento Sócio Econômico: IBOPE. 2000. [S.I. s.n.], 2001

2. BALDRIGHI, S. E. Z. M. et al. A importância do aleitamento natural na prevenção de alterações miofuncionais e ortodônticas. R Dental Press Ortodon Ortop Facial, Maringá, v. 6, n. 5, p. 111-121, set./out. 2001.

3. BARTLEY, M.; BLANE, D.; MONTGOMERY, S. Socioeconomic determinants of health: health and the life course: why safety nets matter. Br Med J, London, v. 314, p.1194-1201, Apr. 1997.

4. CARVALHO.; J. C.; VINKER, F.; DECLERCK, D. Malocclusion, dental injuries and dental anomalies in the primary dentition of Belgian children. Int J Paediatr Dent, London, v. 8, no. 2, p. 137-141, June 1998.

5. ChEVITARESE, A. B.; DELLA, V. D.; MOREIRA, T. C. Prevalence of malocclusion in 4-6 year old Brazilian children. J Clin Pediatr Dent, Birmingham, v. 27, no. 1, p. 81-85, Apr. 2002.

6. CARMINATTI, K. Hábitos deletérios $\mathbf{x}$ maloclusão: nos limites da tipologia facial. 2000. Trabalho de Conclusão de Curso (Especialização)-Centro de Especialização em Fonoaudiologia Clínica, São Paulo, 2000.

7. COSTA, S. et al. Relação oclusal na dentição decídua: estudo pi- 
loto em creches públicas de Salvador. Rev Faculdade Odontol UFBA, Salvador, v. 18, p. 30-34, jul./dez. 1999

8. DAVIS, D. W.; BELL P. A. Infant feeding practices and occlusal outcomes: a longitudinal study. J Can Dent Assoc, Ottawa, v. 57, no. 7, p, 593-594, July 1991

9. DOLCl, G. S.; FERREIRA, E. J. B.; MELLO, A. L. S. F. Relação entre hábitos de sucção e maloclusões. J Brás Ortodon Ortop Facial, Porto Alegre, v. 6, n. 35, p. 379-385, set./out. 2001

10. EMMERICH, A. et al. Relação entre hábitos bucais, alterações oronasofaringianas e mal-oclusões em pré-escolares de Vitória, Espírito Santo, Brasil. Cad Saúde Pública, Rio de Janeiro, v. 20, n. 3, p. 689-697, maio/jun. 2004

11. ENLOW, D. H. Crescimento facial. 3. ed. São Paulo: Artes Médicas, 1993

12. FARSI, N. M. A.; SALAMA, F. S. Sucking habits in Saudi children: prevalence, contributing factors and effects on the primary dentition. Pediatr Dent, Chicago, v. 19, no. 1, p. 28-33, Jan./Feb. 1996;

13. FORTE, F. D. S.; BOSCO, V. L. Prevalência de mordida aberta anterior e sua relação com hábitos de sucção não nutritiva. Pesq Bras Odontop Clin Integr, Santa Catarina, v. 1, n. 1. p. 3-8, jan./abr. 2001

14. FRAZÃO, P. Epidemiologia da oclusão dentária na infância e os sistemas de saúde. 1999. Tese (Doutorado)-Faculdade de Saúde Pública da Universidade de São Paulo, São Paulo, 1999.

15. GRABER, T. M. Ortodoncia: teoria y prática. 3. ed. Trad. José Luis Garcia. México, DF: Nueva Editorial Interamericana, 1972.

16. HANNUKSELA, A.; VÄÄNÄNEN, A. Predisposing factors for malocclusion in 7-year-old children with special reference to atopic desease. Am J Orthod Dentofacial Orthop, St. Louis, v. 92 , no. 4, p. 299-303, Oct. 1987

17. INFANTE, P. F. A epidemiologic study of finger habits in preschool children, as relates to malocclusion, socioeconomic status, race, sex and size of community. J Dent Child, Fulton, v. 43, no. 165 , p. 33-38, jan./feb. 1976

18. IBGE. Manual do recenseador: CD 1.09. Rio de Janeiro, 2000.

19. KATZ, C. R. T. Relação entre hábitos de sucção, mordida aberta anterior, mordida cruzada posterior e morfologia facial em pré-escolares do Recife/PE: um estudo longitudinal. 2003. Tese (Doutorado)-Faculdade de Odontologia da Universidade de Pernambuco, Camaragibe, 2003.

20. KHARBANDA, O. P. et al. A study of the etiological factors associated with the development of malocclusion. J Clin Ped Dent, Birmingham, v. 18, no. 2, p. 95-98, Apr. 1994.

21. KLOCKE, A.; NANDA, R. S.; KAHL-NIEKE, B. Anterior open bite in the deciduous dentition: longitudinal follow-up and craniofacial growth considerations. Am J Orthod Dentofacial Orthop, St. Louis, v. 122, no. 4, p. 353-358, Oct. 2002.

22. LABBOK, M. H.; HENDERSHOT, G. Does breastfeeding protect against malocclusion? An Analysis of the 1981 Child Health Supplement to the National Health Interview Survey. Am J Prev Med, Amsterdam, v. 3, no. 4, p. 227-232, July/Aug. 1987.

23. LANDIS, J. R.; KOCH, G. G. The measurement of observer agreement for categorical data. Biometrics, Amsterdam, v. 33, no. 1, p. 159-174, Mar. 1977

24. LÓPEZ, F.U. et al. Prevalência de maloclusão na dentição decídua. Rev Fac Odontol Porto Alegre, Porto Alegre, v. 42, n. 2, p. 8-1, dez. 2001.

25. MENDES, A. C. R. et al. Associação entre aleitamento, hábitos orais e maloclusões em crianças na cidade de João Pessoa (PB). Rev Odonto Cienc, Porto Alegre, v. 18, n. 42, p. 399-405, out./ dez. 2003.

26. MILAGRE, M. A. Prevalência de oclusopatias na dentição decídua em pré-escolares da cidade de Natal-RN. 2001. TrabaIho de Conclusão de Curso (Especialização)-Associação Brasileira de Odontologia, Rio Grande de Norte, 2001.

27. MOYERS, R. Classificação e terminologia da maloclusão. 4. ed. Rio de Janeiro: Guanabara Koogan; 1991.

28. OLIVEIRA, A. E. Uma transição epidemiológica na oclusão dental em Vitória- ES. 2001. Tese (Doutorado)-Faculdade de Odontologia da Universidade Federal do Rio de Janeiro, Rio de Janeiro, 2001
29. OTUYEMI, O. D et al. Occlusal relationships and spacing or crowding of teeth in the dentitions of 3-4 year-old Nigerian children. Int J Paediatr Dent, Oxford, v. 7, p. 155-160, Sept. 1997.

30. PAUNIO, P.; RAUTAVA, P.; SILLANDAA, M. The finish family competence study: the effects of living conditions on sucking habits in 3 years old, finish children and the association between these habits and dental oclusion. Acta Odontol Scand, Stockholm, v.51, no. 1, p. 23-29, Feb. 1993.

31. PERES, K. G. A. Oclusopatia na dentição decídua: acúmulo de riscos do nascimento à primeira infância. Tese (Doutorado)Faculdade de Saúde Pública, Universidade de São Paulo, São Paulo, 2002.

32. PERIN, P. C. P. Prevalência de má oclusão e necessidade de tratamento ortodôntico, comparando a classificação de Angle e o índice de estética dentária na cidade de Lins. Tese (Doutorado)-Faculdade de Odontologia da Universidade Estadual Paulista, Araçatuba, 2002

33. PINE, C.; PITTS, N. B.; NUGENT, Z. J. British Association for the study of Community Dentistry (BASCD) guidance on sampling for surveys of child dental health. A BASCD coordinated dental epidemiology programme quality standard. Comm Dental Health, [s. I.], v. 14, p. 1-17, 1997. Supplement.

34. POWER, C.; MATTEWS, S.; MANOR, O. Inequalities in self-rated health: explanations from different stages of life. Lancet, Eng land, v. 351, no. 9108, p. 1009-1014, Apr. 1998.

35. ROBLES, F. R. P. et al. A influência do período de amamentação nos hábitos de sucção persistentes e a ocorrência de maloclusões em crianças com dentição completa. Rev Paul Odontol São Paulo, v. 21, n. 3, p. 4-9, maio/jun. 1999

36. SERRA-NEGRA, J. M. C. Aleitamento, hábitos orais deletérios e mal-oclusões existe associação? 1995. Dissertação (Mestrado)-Faculdade de Odontologia da Universidade Federal de Minas Gerais, Belo Horizonte, 1995.

37. SERRA-NEGRA, J. M. C.; PORDEUS, I. A.; ROCHA JUNIOR, J. F. Estudo da associação entre aleitamento, hábitos bucais e maloclusões. Rev Odontol Univ São Paulo, São Paulo, v. 11, n. 2 p. 79-86, abr./jun. 1997.

38. TOMITA, N. E.; BIJELLA, V. T.; FRANCO, L. J. Relação entre hábitos bucais e má oclusão em pré-escolares. Rev Saúde Publica, São Paulo, v. 34, n. 3, p. 299-303, jun. 2000.

39. TSCHILL, P.; WILLIAN, B.; SONKO, A. Malocclusion in the decíduos dentition of Caucasian children. Eur J Orthod, London, v. 19, no. 4, p. 361-367, July/Aug. 1997

40. VALENTE, A.; MUSSOLINO, Z. M. Frequência de sobressaliência, sobremordida e mordida aberta na dentição decídua. Rev Odontol USP, São Paulo, v. 3, n. 3, p. 402-407, jul./set. 1989.

41. LINDEN, F. P. G. M. van der. Genetic and environmental factors in dentofacial morphology. Am J Orthod, St. Louise, v. 8 , no. 52, p. 576-583, Aug. 1966

42. VISCOVIC, R.; VUJANOVIC, M.; BRICIC, V. Prevalence of orthodontic anomalies, analysis and evaluation of dental health in three groups of pre-school children in Zadar. Acta Stomatol Croatica, Yuguslávia, v. 24, no. 4, p. 271-280, Sept. 1990

43. WOON, K. C. Primary dentition occlusion in Chinese, Indian and Malay groups in Malaysia. Aust Orthod J, Brisbane, v. 10, no. 3, p. 183-185, Mar. 1988

44. ZUANON, A. C. C. et al. Relação entre hábito bucal e maloclusão na dentadura decídua. J Brás Odontopediatr Odontol Bebê, São Paulo, v. 3, n. 12, p. 104-108, mar./abr. 2000.

\section{Endereço para correspondência}

Raniere Luiz dos Santos Sousa

Rua Des. Antônio Soares, 1273, Tirol

CEP: 59.022-170 - Natal/RN

E-mail: raniere@clinicavicentedepaula.com.br 